\title{
Black Urine and Black Pleural Fluid: A Distinctive Presentation of Metastatic Melanoma
}

\author{
Samer Mishe'el ${ }^{1}$, Michael Ziv ${ }^{2}$, Naiel Bisharat ${ }^{1,3}$ \\ ${ }^{1}$ Department of Medicine D, Emek Medical Center, Afula, Israel \\ ${ }^{2}$ Department of Dermatology, Emek Medical Center, Afula, Israel
}

${ }^{3}$ The Ruth and Bruce Rappaport Faculty of Medicine, Technion-Israel Institute of Technology, Haifa, Israel

\section{Doi: 10.12890/2016_000416 - European Journal of Case Reports in Internal Medicine - ๑ EFIM 2016}

\begin{abstract}
Received: $23 / 02 / 2016$
Accepted: 01/03/2016

Published: 07/04/2016
\end{abstract}

How to cite this article: Mishe'el S, Ziv M, Bisharat N. Black urine and black pleural fluid: a distinctive presentation of metastatic melanoma. EJCRIM 2016;3:doi:10.12890/2016_000416

Conflicts of Interests: The Authors declare that there are no competing interests.

This article is licensed under a Commons Attribution Non-Commercial 4.0 License

\section{ABSTRACT}

Metastatic melanoma is an uncommon clinical entity which can have an unusual presentation. We describe a patient with metastatic melanoma who presented with diffuse melanosis cutis, black urine and black pleural effusion. Very few medical conditions can cause black discoloration of body fluids, so this should prompt physicians to search for a number of potential underlying causes.

\section{LEARNING POINTS}

- Diffuse melanosis cutis is a rare presentation of metastatic melanoma.

- Malignant melanoma with thoracic metastases can cause a black pleural effusion.

- Physicians should be aware of potential causes of black discoloration of body fluids.

\section{KEYWORDS}

Melanoma; pleural effusion; diffuse melanosis cutis; black urine.

\section{INTRODUCTION}

Malignant melanoma is the most serious of the skin cancers and has shown an increasing incidence over the past 30 years ${ }^{[1]}$. Melanoma can present at any stage from a single localized cutaneous lesion up to rapidly invasive disease affecting multiple organs. Diffuse melanosis cutis $(D M C)$ is a rare complication in patients with metastatic melanoma ${ }^{[2]}$. We describe a patient who presented with weight loss, worsening dyspnoea, slate-grey skin discoloration and black urine caused by metastatic melanoma with DMC. The case report describes the patient's course and discusses this atypical presentation.

\section{CASE REPORT}

A 77-year-old man was referred to the emergency department due to worsening dyspnoea and weight loss. The patient mentioned that he had experienced dyspnoea for the previous month which worsened in the week before his admission. He also complained of an unintentional weight loss of $12 \mathrm{~kg}$ within a 2-month period and had noticed a bluish-grey discoloration of his face, hands and abdomen. In addition, he had observed a black discoloration of the urine in the week before his visit.

His medical history was remarkable for ischaemic heart disease, congestive heart failure, hypertension, diabetes mellitus and benign prostatic enlargement, which were treated with beta-blockers, calcium channel blockers, diuretics, insulin and acetylsalicylic acid. 
His physical examination was remarkable for black discoloration of the face and slate-grey discoloration of the hands and abdomen (Fig. 1). A chest x-ray showed bilateral pleural effusion. A urine specimen was initially dark yellow but turned black after exposure to air at room temperature (Fig. 2). A chest and abdominal computed tomography scan confirmed the presence of bilateral pleural fluid, hepatomegaly with a large amount of peritoneal fluid, and mediastinal, retroperitoneal and inguinal lymphadenopathy. Bedside ultrasound-guided thoracentesis was performed with drainage of $1,500 \mathrm{ml}$ of an opaque yellow-coloured pleural fluid that turned black after exposure to room air (Fig. 3).

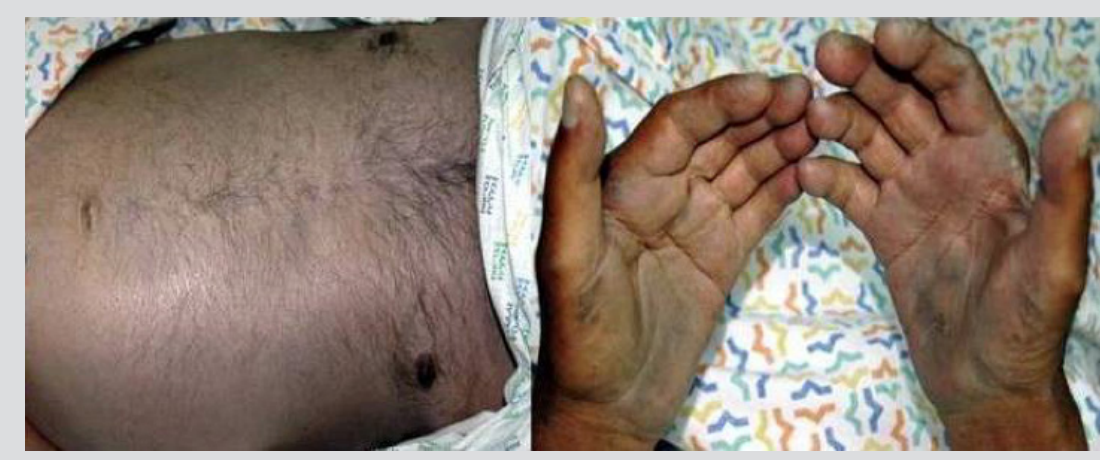

Figure 1: Slate-grey discoloration of the skin on the abdomen and the palms of the hands.

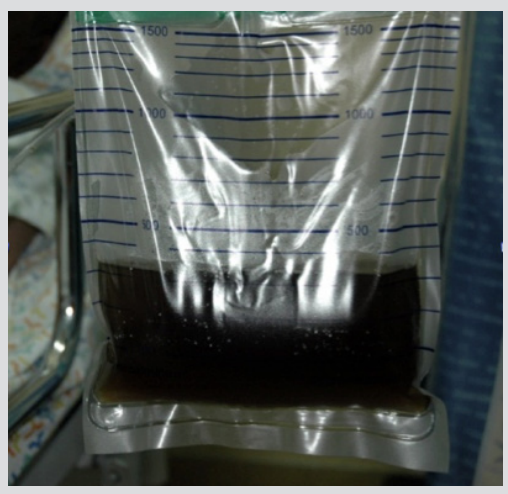

Figure 2: Urine bag showing black discoloration of urine after exposure to room air.

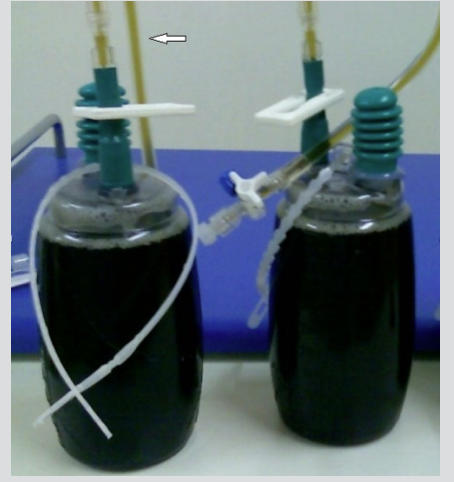

Figure 3: Black discoloration of pleural fluid; note the dark yellow colour of the fluid in the connecting tubes (white arrow)

Centrifugation produced a yellow supernatant and black sediment. Examination of the fluid was consistent with an exudative pleural effusion (pH 7.1, glucose $22 \mathrm{mg} / \mathrm{dl}$, lactate dehydrogenase $1622 \mathrm{U} / \mathrm{l}$, protein $3.9 \mathrm{~g} / \mathrm{dl}$ ). Intra-pleural levels of amylase were not elevated. Gram stain, and bacterial and fungal cultures of the pleural fluid were negative. Pleural fluid cytology revealed numerous neoplastic cells with cytoplasmic melanin pigment deposits in Diff-Quick stain, consistent with metastatic melanoma. Urine cytology showed tubular cells with brown pigment that was positive for Fontana stain, indicating that the black urine was caused by melanin pigment; no malignant melanoma cells were seen in the sample. A skin biopsy taken from hyperpigmented areas on the abdomen showed minimal perivascular lymphocytic infiltrate in the upper dermis, while Schmorl's staining demonstrated scattered melanin-laden histiocytes in the dermis and underlying subcutis. Immunohistochemical staining did not show cutaneous melanoma metastases in the biopsy specimens. The patient's condition quickly deteriorated and he died after 2 weeks. The patient's family refused an autopsy. 


\section{DISCUSSION}

The patient died of metastatic melanoma with DMC. The striking features of this case are the skin discoloration and the black colour of the urine and pleural fluid. DMC is considered an extremely rare presentation of metastatic melanoma, and the exact pathophysiology underlying its appearance is unclear. It has been suggested that melanin precursors, melanin and melanosomes liberated by cytolytic metastatic melanoma deposits are phagocytosed by dermal histiocytes, manifesting clinically as diffuse melanosis ${ }^{[2]}$. DMC is associated with a poor prognosis with an estimated mean survival of $\sim 4$ months for affected patients ${ }^{[2]}$.

Black pleural effusion is an uncommon entity that has been rarely discussed in the literature. A recent review addressed the issue and detailed the possible differential diagnosis ${ }^{[3]}$. The authors identified four possible aetiologies: infection associated with Aspergillus niger and Rhizopus oryzae, malignant cells containing melanin pigment associated with melanoma, haemorrhage and haemolysis associated with nonsmall cell lung cancer or rupture of a pancreatic pseudocyst, and other causes including charcoal-containing empyema and crack cocaine inhalation with intracellular or extracellular carbon pigment transport across the visceral pleura ${ }^{[4]}$.

Likewise, black urine is an extremely rare entity that has been rarely discussed. Black urine, termed melanuria, is an uncommon finding in patients with disseminated melanoma ${ }^{[5]}$. In our case, other causes of dark urine such as gross haematuria, alkaptonuria, porphyria and rhabdomyolysis were either ruled out or were considered highly unlikely given the above findings. In melanuria, as well as in black pleural effusion, the urine is yellow and darkens as the colourless melanin precursors oxidize in the presence of air ${ }^{[5]}$.

Although rarely described, black discoloration of body fluids is a unique and rather puzzling challenge. Very few medical conditions can cause black discoloration of body fluids, which should prompt physicians to search for a number of potential underlying causes.

\section{REFERENCES}

1. Chen ST, Geller AC, Tsao H. Update on the epidemiology of melanoma. Curr Dermatol Rep 2013;2:24-34

2. Sebaratnam DF, Venugopal SS, Frew JW, McMillan JR, Finkelstein ER, Martin LK, et al. Diffuse melanosis cutis: a systematic review of the literature. J Am Acad Dermatol 2013;68:482-488.

3. Saraya T, Light RW, Takizawa H, Goto H. Black pleural effusion. Am J Med 2013;126:641.e1-6.

4. Singh B, Greenebaum E, Cole R. Carbon-laden macrophages in pleural fluid of crack smokers. Diagn Cytopathol 1995;13:316-319.

5. Santos AL, Ibrahim G, Mount S, Thomas C, Weise WJ. The case: a Caucasian male with dark skin, black urine, and acute kidney injury. Kidney Int 2009;76:1295-1296. 